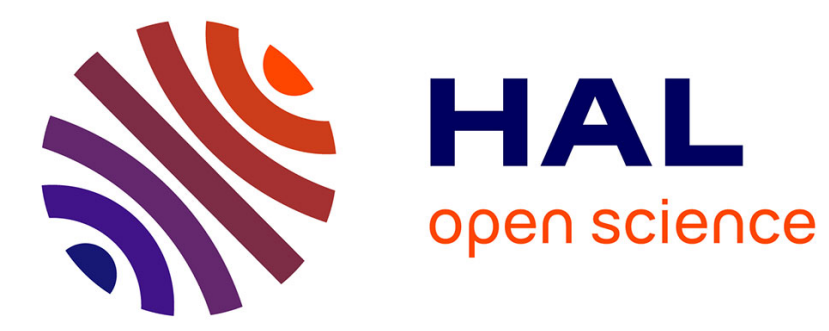

\title{
New approaches to supercontinuum control in the long pulse regime
}

\author{
G. Genty, J.M. Dudley
}

\section{To cite this version:}

G. Genty, J.M. Dudley. New approaches to supercontinuum control in the long pulse regime. Optical components and materials VI, Jan 2009, San Jose, United States. 10.1117/12.814721 . hal-00476101

\section{HAL Id: hal-00476101 \\ https://hal.science/hal-00476101}

Submitted on 14 May 2021

HAL is a multi-disciplinary open access archive for the deposit and dissemination of scientific research documents, whether they are published or not. The documents may come from teaching and research institutions in France or abroad, or from public or private research centers.
L'archive ouverte pluridisciplinaire HAL, est destinée au dépôt et à la diffusion de documents scientifiques de niveau recherche, publiés ou non, émanant des établissements d'enseignement et de recherche français ou étrangers, des laboratoires publics ou privés. 


\title{
New approaches to supercontinuum control in the long pulse regime
}

\author{
G. Genty and J M. Dudley ${ }^{\mathrm{b}}$ \\ ${ }^{a}$ Department of Physics, Tampere University of Technology, Tampere, Finland \\ bInstitut FEMTO-ST, Université de Franche-Comté, Besançon, France
}

\begin{abstract}
We review recent developments for controlling supercontinuum spectral structure generated in photonic crystal fibers. Specifically, we describe two different approaches to generating supercontinuum with improved stability characteristics in the long pulse regime where the use of picosecond or nanosecond pulses is generally associated with large instabilities and decoherence. On the one hand, the use of dispersion-varying fibers results in efficient soliton compression and symmetric dispersive wave generation mechanism leading to near-uniform spectra. On the other hand, applying a modulation on the input pulse allows control of "optical rogue wave" fluctuations with significant stabilization of the long wavelength Raman solitons position.
\end{abstract}

Keywords: Optical fibers, supercontinuum, soliton, modulation instability

\section{INTRODUCTION}

Following its first observation by Ranka et al. in 1999-2000 $0^{[1]}$, supercontinuum (SC) generation in photonic crystal fiber has remained a subject of intense research. Motivated by important applications in precision frequency metrology, initial effort focussed on developing a clear physical understanding of the underlying mechanisms and noise properties of SC generation seeded by femtosecond pump pulses, a regime which is now very well understood ${ }^{[2-5]}$. Research is now shifting towards detailed studies of the dynamical properties for cases where spectral broadening is initiated in the socalled "long pulse" regime, using picosecond to nanosecond pulses, or even a continuous wave pump, as SC can be generated using a variety of compact and relatively inexpensive pump sources. In fact, under these conditions and pumping in the anomalous dispersion regime, the SC spectral broadening has been shown to be associated with a very rich range of dynamical behavior, involving spontaneous pulse break up due to modulation instability (MI) followed by the propagation and interaction between very large numbers of ejected soliton pulses ${ }^{[5-13]}$. With such long pulses, however, the generated SC typically suffers from large shot-to-variations resulting from noise-seeded MI and this can limit the potential application of the generated broadband spectra. There is thus significant interest in developing techniques that allow a degree of control into the shaping and stability of SC generation in this regime. Furthermore, crucial insight into the mechanisms underlying SC fluctuations has recently been provided by Solli et al. who used a novel wavelength to time conversion technique to directly measure the statistics of the shot to shot SC noise ${ }^{[14]}$. Of particular interest is the fact that fluctuations in the spectral structure of the SC in the long pulse regime were shown to be associated with the generation of "optical rogue waves" -statistically rare events associated with the extreme red shift of Raman solitons pulses on the long wavelength SC edge ${ }^{[14-16]}$. Here, we explore different routes to limit the statistical fluctuations associated with SC generation in the long pulse regime.

Significantly, detailed previous studies have suggested that fibers with longitudinally varying dispersion could be advantageous in generating flatter and broader SC spectra compared to fibers with uniform longitudinal dispersion ${ }^{[17-23]}$. In particular, fibers with a decreasing dispersion profile were found to produce uniform and wide SC with a high degree of coherence useful for pulse compression applications. Initial studies have focussed on low peak pulses thereby resulting in SC generation with limited bandwidth and spectral density. Revisiting the use of such fibers for SC generation we show that it is also possible to generate octave-spanning SC from high peak power pulses yet with high stability characteristics. The advantage of such pumping scheme lies in the efficient higher-order soliton compression and dispersive wave generation which precludes MI and associated statistical fluctuations ${ }^{[24]}$.

Another approach consists in controlling the generation of rogue waves inherent to the long pulse regime with anomalous pumping ${ }^{[14-16]}$. As a matter of fact, it has been shown that a degree of enhancement of the rogue wave generation can be obtained through the use of a small amplitude modulation on the input pulse envelope $\mathrm{e}^{[15-16]}$. We extend 
this concept of rogue wave control to examine in more detail the degree to which the structure and stability of the output SC spectra can be modified through a suitable input pulse modulation and provides practical guidelines that can lead to a fully coherent continuum.

\section{SUPERCONTINUUM GENERATION IN DISPERSION DECREASING FIBERS}

The objective of this section is to address SC generation in fibers with longitudinally-varying convex dispersion profile (DF-DDF) which typically exhibits two zero-dispersion wavelengths whose separation reduces with distance (see Fig. 1). In this type of fiber, the dispersion at the pump wavelength changes from anomalous at the input end to normal at the output end. Fibers with two ZDWs have been demonstrated in the early 1980's using double clad fiber technolog ${ }^{[25-26]}$ and it was in fact the use of such "dispersion-flattened" fibers with longitudinally decreasing dispersion that originally led to the extensive studies of SC generation at telecommunications wavelengths. A major result of these studies was the statement of clear fiber design criteria for the generation of a uniform SC spectra: (i) a dispersion profile presenting decreasing anomalous GVD with propagation and (ii) a dispersion-flattened convex wavelength variation of the GVD exhibiting two zero dispersion wavelengths at the fiber input and centered near the pump ${ }^{[23]}$.

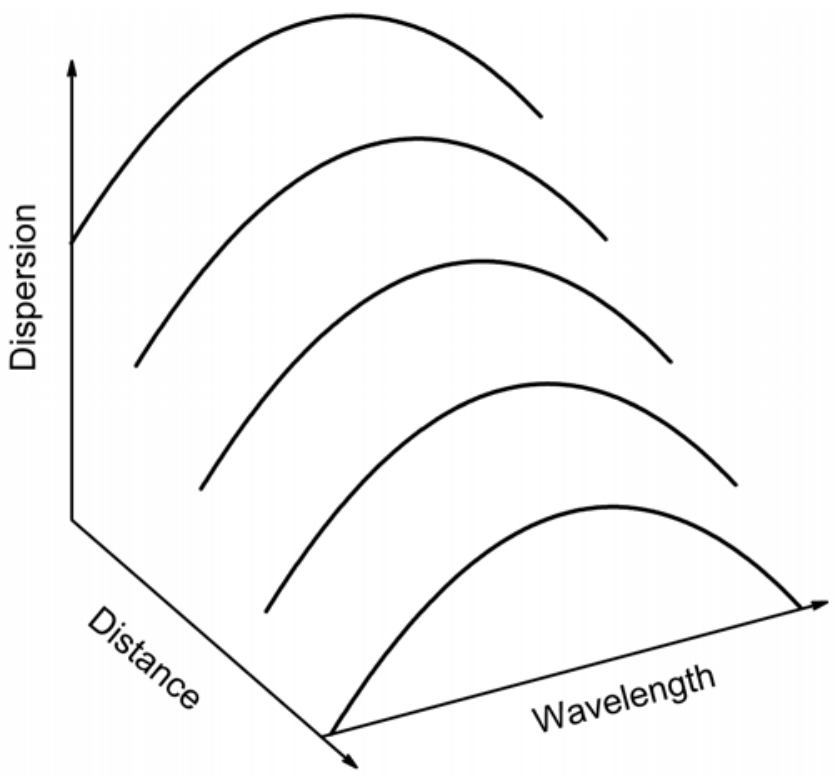

Fig. 1. Convex dispersion profile of a dispersion-decreasing fiber.

\subsection{Basic dynamics of Sc generation in DF-DDF}

Our numerical model uses a generalized propagation equation suitable for studying broadband pulse evolution in optical fibers $^{[5]}$

$$
\frac{\partial A}{\partial z}-\sum_{k \geq 2} \frac{i^{k+1}}{k !} \beta_{k} \frac{\partial^{k} A}{\partial t^{k}}=i \gamma\left(1+i \tau_{\text {shock }} \frac{\partial}{\partial t}\right)\left(A(z, t) \int_{-\infty}^{+\infty} R\left(t^{\prime}\right)\left|A\left(z, t-t^{\prime}\right)\right|^{2} d t^{\prime}\right) .
$$

Here, $A=A(z, t)$ is the electric field envelope, the $\beta_{k}$ 's are the fiber dispersion coefficients at center frequency $\omega_{0}$, and $\gamma=$ $n_{2} \omega_{0} /\left(c A_{\text {eff }}\right)$ is the nonlinear coefficient, with $n_{2}=3.2 \times 10^{-20} \mathrm{~m}^{2} / \mathrm{W}$ the nonlinear refractive index and $A_{\text {eff }}$ the fiber effective area evaluated at $\omega_{0}$. The time derivative term describes effects such as self-steepening and optical shock formation, characterized by a timescale $\tau_{\text {shock }}=1 / \omega_{0}$. In the context of fiber propagation, the frequency dependence of the modal effective area $A_{\text {eff }}$ can introduce significant dispersion in the nonlinear response, and this can be included to a very good approximation through a first order correction to $\tau_{\text {shock }}{ }^{[27]}$. The nonlinear response $R(t)=\left(1-f_{\mathrm{R}}\right)(t)+f_{\mathrm{R}} h_{\mathrm{R}}(t)$ with $f_{\mathrm{R}}=$ 0.18 includes instantaneous and Raman contributions. The response $h_{\mathrm{R}}$ is determined from the experimental fused silica 
Raman cross-section, but similar results can be obtained using analytic approximations for the Raman term. Noise is included in the frequency domain through a one photon per mode background, and via the term $\Gamma_{\mathrm{R}}$ which describes thermally-driven spontaneous Raman scattering ${ }^{[5]}$.

In order to illustrate the specific dynamics and characteristics of SC generation in DF-DDF we first examine low power SC generation regime with parameters typical of those used in previous studies ${ }^{[23]}$. We consider input pulses at $1550 \mathrm{~nm}$ with peak power $P_{0}=1 \mathrm{~W}$ and duration (FWHM) of $\Delta \tau=5$ ps propagating in a $1 \mathrm{~km}$ length of DF-DDF. The input pulse parameters correspond to an average power of $50 \mathrm{~mW}$ at a repetition rate of $10 \mathrm{GHz}$, typical of amplified pulses from high-repetition rate harmonically-modelocked fiber lasers. The dispersion coefficients are calculated from a longitudinally varying dispersion model given by

$$
D(z)=D_{0}\left(1-\frac{z}{L_{0}}\right)+\frac{D_{2}}{2}\left(\lambda-\lambda_{0}\right)^{2}
$$

with $D_{0}=6 \mathrm{ps} \mathrm{nm}^{-1} \mathrm{~km}^{-1}, D_{2}=-2 \times 10^{-4} \mathrm{ps} \mathrm{nm}^{-3} \mathrm{~km}^{-1}$, and $L_{0}=900 \mathrm{~m}$. In this model the parameter $D_{2}$ sets the convexity of the profile while $L_{0}$ represents the distance at which the dispersion changes from anomalous to normal at the pump wavelength. Figure 2 plots the dispersion parameter $D$ at selected distances to illustrate the longitudinal evolution of the convex dispersion from anomalous to normal values in the vicinity of the pump. For values of $z<L_{0}$, the profile presents two ZDWs whose separation decreases with distance. At distances exceeding $L_{0}$, the dispersion is normal at all wavelengths. It is assumed that the pump wavelength corresponds to $\omega_{0}$, and the nonlinear coefficient of the fiber is assumed to be constant $\gamma=5 \mathrm{~W}^{-1} \mathrm{~km}^{-1}$. For the pump wavelength consider here $\tau_{\text {shock }}=0.822 \mathrm{fs}$.

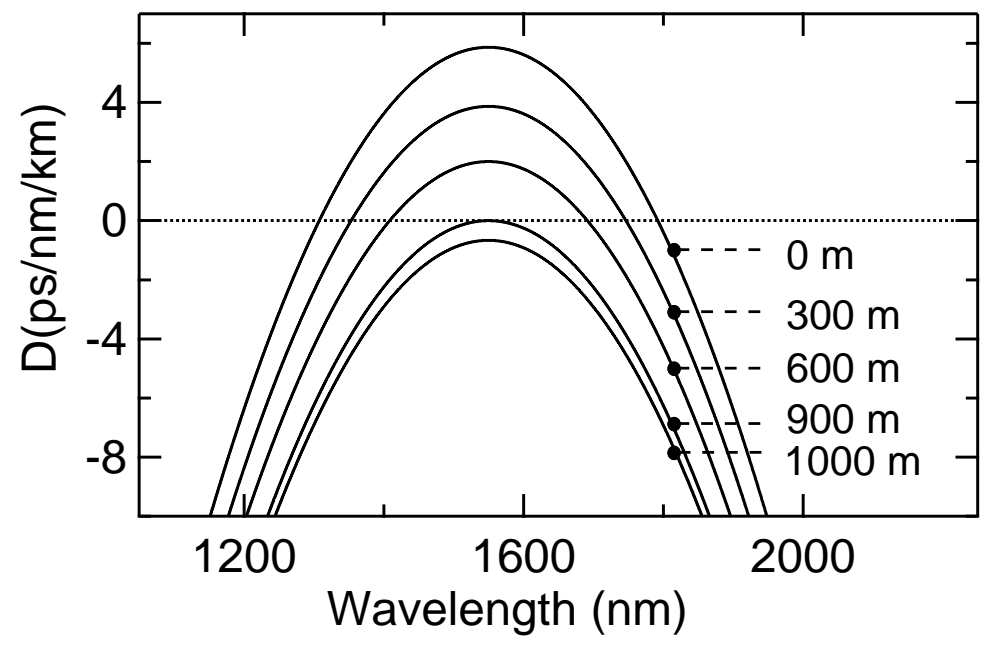

Fig. 2. Dispersion profile for the DF-DDF as described in the text at selected fiber lengths. The zero-line is shown to highlight the variation in the separation between the two ZDWs with distance ${ }^{[24]}$.

Figure 3 shows the spectral and temporal evolution observed in this case. The dynamics of the SC formation in a DFDDF differs substantially from that observed in a fiber with decreasing zero-dispersion wavelength. The initial pump pulse here corresponds to a second-order soliton $N=2.3$, and the adiabatically decreasing dispersion combined with the Kerr nonlinearity induces an initial phase of symmetrical spectral broadening and associated temporal compression. Such adiabatic pulse compression in dispersion-decreasing fibers has been analyzed originally for injected fundamental solitons ${ }^{[28-29]}$ but higher-order soliton compression in these fibers leads to similar dynamics except for a much reduced optimum compression distance ${ }^{[30]}$. This initial stage is in fact qualitatively identical to the case of fiber with decreasing zero-dispersion wavelength. 


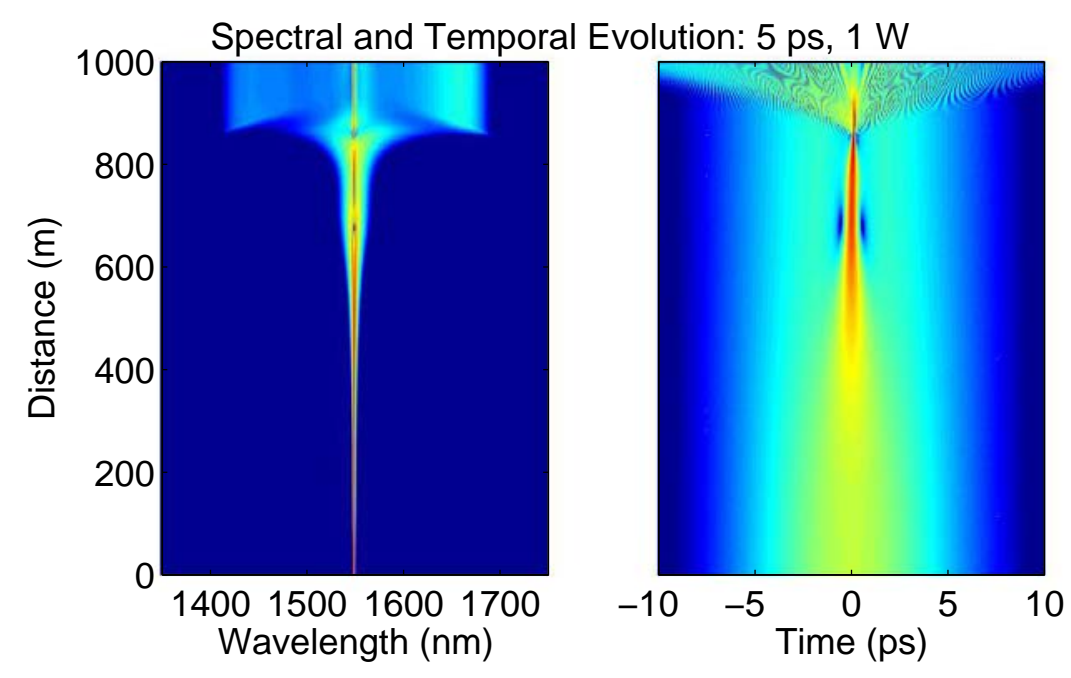

Fig. 3. Evolution of spectral (left) and temporal intensity (right) for a 5 ps duration, $1 \mathrm{~W}$ peak power pulse at $1550 \mathrm{~nm}$ propagating in a DF-DDF ${ }^{[24]}$ as described in the text.

This phase of initial compression can be more conveniently visualized by plotting the evolution of the key pulse parameters along the length of the DF-DDF as is shown in Fig. 4(a). Specifically, it can be seen how the smooth increase in peak power and decrease in pulse duration are associated with the initial evolution of the propagating pulse towards a fundamental soliton. It is this adiabatic evolution in the soliton parameters that precludes the break-up of the pulse due to soliton fission otherwise observed in SC generation. The point of maximum compression is reached slightly before the propagation distance of $L_{0}$. At this point the spectrum experiences significant broadening into the normal dispersion regions. The initial phase of propagation is associated with adiabatic compression and spectral broadening but, after a propagation distance of around $850 \mathrm{~m}$, the spectral bandwidth has increased sufficiently to induce dispersive wave generation symmetrically with respect to the pump wavelength. In the case of a fiber with a single ZDW, dispersive wave generation is associated with a modification of the pump spectrum due to spectral recoil, but the simultaneous generation of two dispersive waves in the fiber considered here leads to opposite spectral recoils which balance. This prevents any shift in the spectrum of the pump and contributes to yield a continuous spectrum from the edges towards the center. For propagation beyond $L_{0}$ the bandwidth stabilizes somewhat while the temporal intensity broadens and develops strong modulation. The slight asymmetry observed in the output spectra arises from stimulated Raman scattering.

Besides the symmetrical aspect of the spectrum, another noteworthy difference with SC generation in other types of fibers is the apparent flatness of the SC spectrum. Two factors principally influence the flatness of the spectrum. Firstly, although the phase-matching condition for dispersive wave generation is intrinsically narrowband, the longitudinally varying separation between the pump and the ZDWs results in a continuous and broadband range of phase-matched wavelengths. Secondly, after propagating beyond $L_{0}$ when the dispersion is normal everywhere, the residual pump components temporally broaden and overlap with the frequency-shifted dispersive waves, facilitating interaction through cross-phase modulation. The combined result of these dynamics is a broad and flat SC almost free of fine structure. The residual spectral structure at the pump wavelength is a typical feature of higher-order solition compression, and corresponds to the broad time-domain pedestal. The absence of soliton fission would suggest that this propagation regime would be highly coherent. Indeed, this is the case, and is shown explicitly in Fig. 4(b) based on an ensemble of 20 simulations performed with different random noise seeds. There is clearly negligible pulse-to-pulse intensity noise, and near perfect coherence across the SC bandwidth. 
(a) Longitudinal Evolution

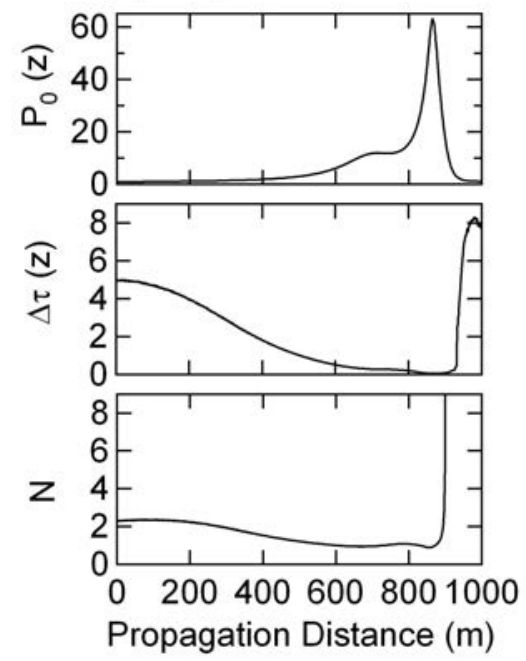

(b) Output Spectral Characteristics

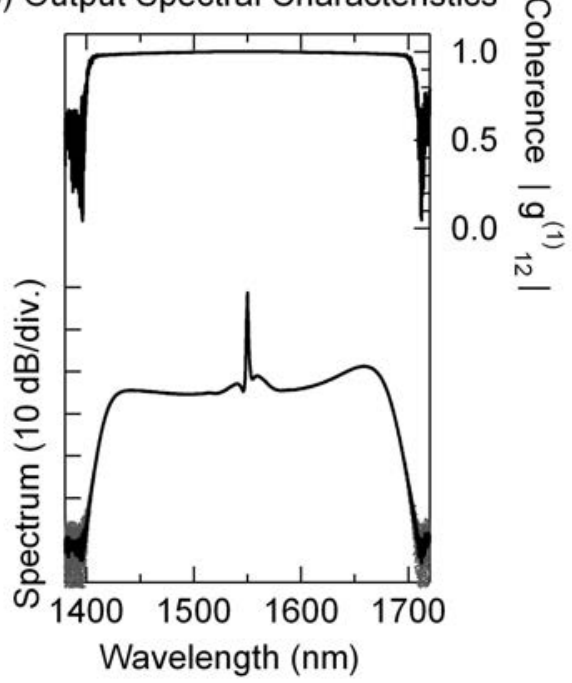

Fig. 4. Further details of the dynamical evolution shown in Fig. $3^{[24]}$. (a) shows the longitudinal evolution from one simulation of the peak power $P_{0}(\mathrm{z})$, pulse duration (FWHM) $\Delta \tau$ and (for propagation distance $z<L_{0}$ the associated soliton order. (b) Results from 20 simulations showing the output spectra (bottom curves, left axis) and corresponding degree of coherence (top curve, right axis). For the spectral plots, the gray curves show the individual spectra from the ensemble while the solid line shows the calculated mean. The difference is apparent only in the spectral wings.

Aspects of these specific dynamics can be better visualized in the projected-axis spectrogram ${ }^{[31]}$ plotted in Fig. 5 . The specific dispersion profile of the fiber manifests itself in the fact that the dispersive waves are spread in time-frequency space in an $S$-like structure. A careful inspection of the spectrogram also reveals the physical origin of the strong temporal modulation in the pulse wings as arising from the beating of the unconverted and dispersed pump and the dispersive wave components. The spectrogram also clearly reveals how the unconverted pump is sufficiently temporally broadened to overlap with all the dispersive wave components. Of additional interest is the fact that the $S$-like characteristic of the spectrogram leads to a temporal chirp of this intensity modulation (see the expanded portion of the intensity profile). 

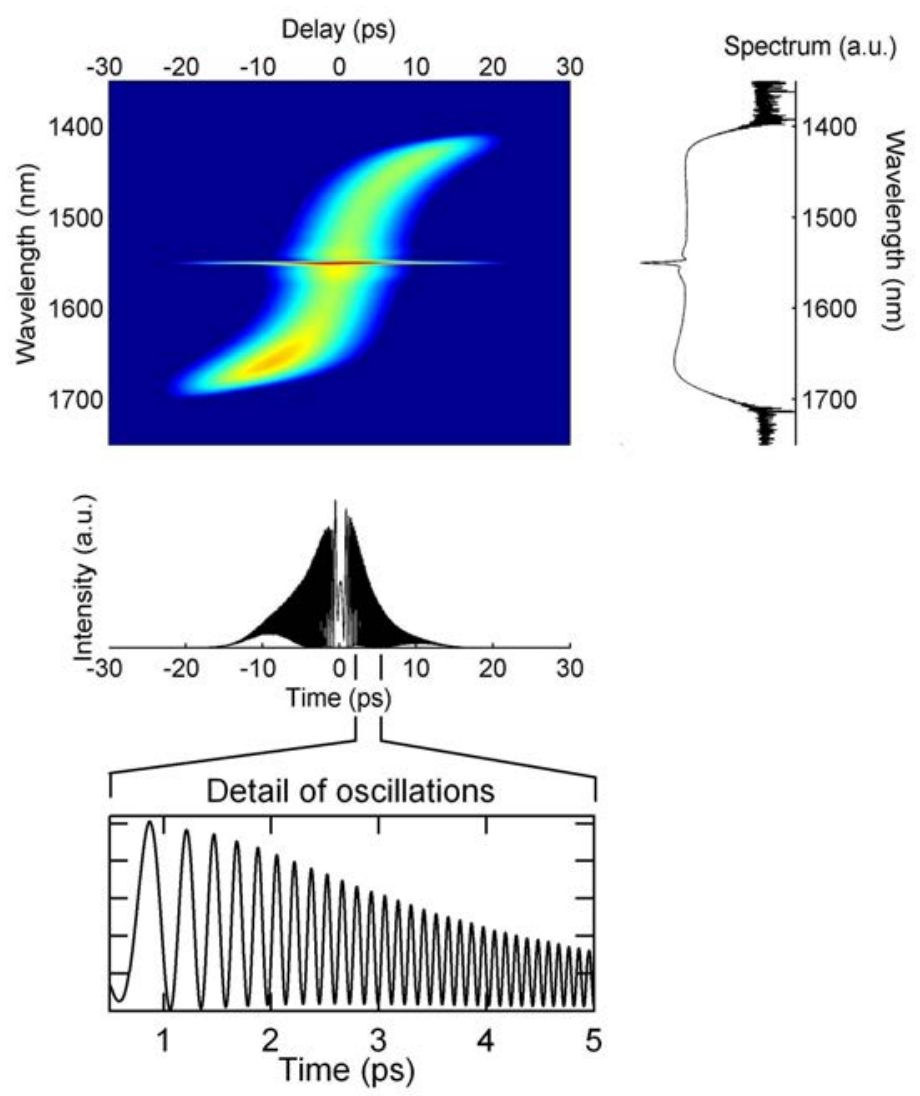

Fig. 5. Projected axis spectrogram of the output pulse of Fig. 3 illustrating how the flattened spectrum is associated with a complex modulated temporal structure ${ }^{[24]}$. The gate function used in the spectrogram calculation had duration

(FWHM) of $\Delta \tau=1 \mathrm{ps}$, the same as the initial pump pulse.

\subsection{SC generation in DF-DDF with larger input peak power}

Although propagation in DF-DDF was initially studied in the context of low power SC generation for telecommunications applications, suitable scaling of the fiber dispersion can lead to the similar generation of broadband flat SC with input pulses over a much wider parameter range. Therefore, DF-DDF can be envisaged as a more general route towards SC generation of picosecond pulses, with precise fiber designs yielding coherent SC generation at peak power levels in the hundreds of Watts range and beyond. Two examples of such designs yielding coherent SC around $1550 \mathrm{~m}$ with significantly higher peak power than considered above are displayed in Fig. 6.

The first example consider $200 \mathrm{~W}$ peak power pulses of duration (FWHM) 1 ps propagating in a $7 \mathrm{~m}$ length of DF-DDF with $D_{0}=10 \mathrm{ps} \cdot \mathrm{nm}^{-1} \cdot \mathrm{km}^{-1}, D_{2}=-2 \times 10^{-4} \mathrm{ps} \mathrm{nm}^{-3} \mathrm{~km}^{-1}, L_{0}=6 \mathrm{~m}$, and $\gamma=5 \mathrm{~W}^{-1} \mathrm{~km}^{-1}$. This yields similar spectral characteristics as with low input soliton order, again with excellent coherence. The input soliton order is in this case $N=5.0$. Results for $1200 \mathrm{~W}$ peak power pulses of duration (FWHM) 1 ps propagating in a $1.4 \mathrm{~m}$ length of DF-DDF with $D_{0}=30 \mathrm{ps} \mathrm{nm}^{-1} \mathrm{~km}^{-1}, D_{2}=-2 \times 10^{-4} \mathrm{ps} \mathrm{nm}^{-3} \mathrm{~km}^{-1}, L_{0}=1.2 \mathrm{~m}$, and $\gamma=5 \mathrm{~W}^{-1} \mathrm{~km}^{-1}$ are also shown in Fig. 6. Although we do not see the same degree of spectral flatness at this very high power level, we can still observe broadband spectral generation. The input soliton order here is $N=7.1$. Also shown in these figures is the corresponding evolution of the soliton parameters along the fiber to illustrate the similar smooth evolution dynamics to the low power case in Fig. 4. In fact the trend observed in the SC generation for increased input soliton order is rather general, which allows to give some guidelines that will typically result in a broadband coherent SC. For larger $N$ the propagation distance and $L_{0}$ should be reduced while the dispersion at the pump wavelength at the fiber input should be increased. Smaller convexity parameter of the dispersion profile generally yields better coherence at it prevents soliton fission but at the expenses of the bandwidth which is determined by the phase-matching condition of the DW. Also, and as is usually the case with SC 
generation in the anomalous pumping regime, a soliton order less than $N=10$ calculated at the DF-DDF input ensures high coherence and stability, larger input values yielding good stability only for very large values $D_{2}$.

(a) $1 \mathrm{ps}, 150 \mathrm{~W}$
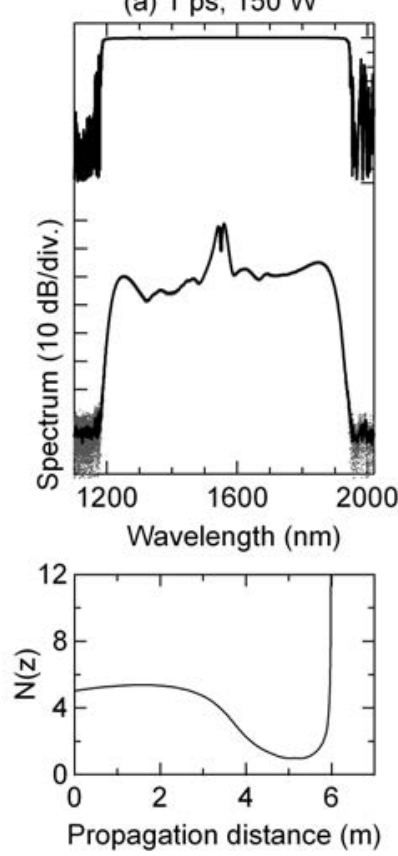

(b) $1 \mathrm{ps}, 1.2 \mathrm{~kW}$

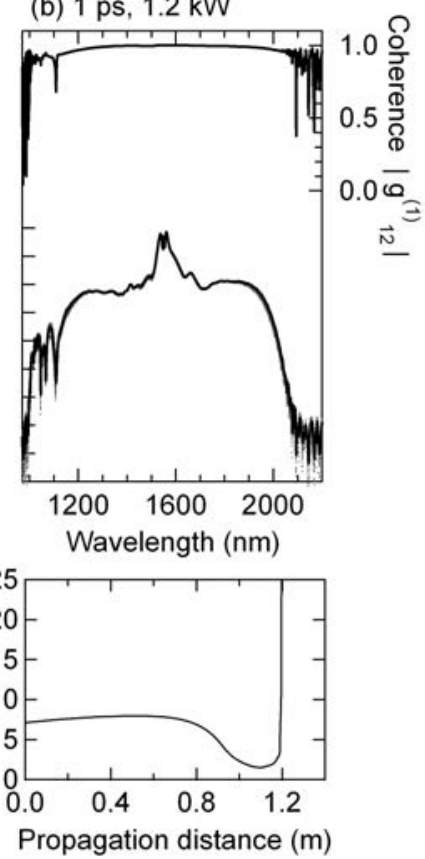

Fig. 6. Results showing evolution in DF-DDF of pulses ${ }^{[24]}$ with (a) 1 ps duration and $150 \mathrm{~W}$ peak power and (b) 1 ps duration and $1.2 \mathrm{~kW}$ peak power. The top figures present results from 20 simulations showing the output spectra (bottom curves, left axis) and corresponding degree of coherence (top curve, right axis). For the spectral plots, the gray curves show the individual spectra from the ensemble while the solid line shows the calculated mean, although the difference is apparent only in the wings of the spectra. The bottom curves in each case show the evolution of the soliton number $N(\mathrm{z})$ with propagation for distances less than $L_{0}$.

A significant potential application of the flattened SC spectra generated in DF-DDF is in the field of pulse compression. The initial nonlinear evolution phase in DF-DDF has previously been studied in detail to directly yield a temporally compressed pulse. In this case, the compressed pulse is accompanied by a very broad low amplitude pedestal spanning over several picoseconds. The extension of the initial propagation into the SC generation regime is expected to yield vastly improved compressed pulse quality. Indeed, although the output temporal pulse in this case is complex and could not be exploited directly, the high coherence across the spectrum would, however, be expected to lead to very high quality compressed pulses after appropriate spectral phase compensation ${ }^{[32-33]}$.

We note that the dispersion parameters used in the simulations presented in this section are within the realistic range of what might be expected with recent advances in dispersion-flattened and PCF technology around $1550 \mathrm{~nm}$. Comparable work has also explored the development of PCF with dual ZDWs centered around $1 \mu \mathrm{m}$ so that exploring tapered PCF design in this wavelength range could provide further possibilities for coherent SC generation for longer pulses with $\mathrm{Yb}^{3+}$ based sources. When considering tapered PCF, however, the ideal dynamics described above may be modified by the variation in effective area along the propagation direction ${ }^{[36]}$.

\section{SUPERCONTINUUM GENERATION WITH INPUT MODULATION}

Studies in the femtosecond regime have shown how the generated SC spectra can be controlled by varying the input conditions in a more sophisticated manner, using chirped input pulses to modify the propagation dynamics, using dual frequency femtosecond pumping to induce cascaded four wave mixing, or using femtosecond envelope modulation to 
influence the Raman soliton noise properties. In the long-pulse long pulse regime, modified initial conditions can also significantly influence the output SC characteristics and stability. For example, Solli et al. numerically demonstrated a correlation between rogue soliton pulse height and a low-amplitude low amplitude localized noise burst on the leading edge of the picosecond pump pulses. This idea was extended in Ref. 15, where numerical simulations were again used to show that modified rogue soliton statistics could be observed using a $4 \%$ intensity modulation across the full extent of the pulse envelope. Furthermore, s recent eprint by Solli et al. has reported significant experimental results, showing that seed modulation of SC generation at the $-30 \mathrm{~dB}$ level can also introduce an effective phase transition in the SC stability. The dramatic effects of these modified input conditions illustrate the sensitivity of the initial MI propagation phase of long-pulse long pulse SC generation to coherent input modulation. Another study has examined the potential parameter space of input modulation more extensively, focussing on how both the SC spectral intensity and stability are modified as functions a function of the modulation parameters. A major result was the discovery that certain modulation parameters yield stabilized stabilized spectra with Raman soliton peak power statistics transformed from an "L-shaped" extreme-value distribution to a near-Gaussian near-gaussian distribution with significantly reduced peak power fluctuations below $5 \%$.

Here, we use physical arguments and numerical simulations to examine in detail the degree to which stability of the output SC spectra can be modified through a suitable input pulse modulation. Our major result is that the use of relatively large modulation strength on the input pulse can lead to a very high degree of coherence of the generated supercontinuum provided the modulation frequency and fiber length are chosen adequately. Although the use of such strongly modulated fields has been applied in previous studies of beat signal reshaping and induced modulation instability, the results here are the first that examine applications in supercontinuum spectral shaping and stability. This technique could have high impact in the spectral stabilization and control of SC generation in the long pump pulse regime.

\subsection{Physical guidelines}

A temporal modulation is imposed across the entire pump pulse envelope in a standard way by adding to the input field a replica downshifted by $f_{\mathrm{m}}$ such that the total field can be written $A_{\mathrm{m}}(t)=A(0, t) \times\left[1+a_{0} \exp \left(\mathrm{i} 2 \pi f_{\mathrm{m}} t\right)\right]$. Here $A(0, t)=\sqrt{ } P_{0}$ $\exp \left(-2 \ln 2 \mathrm{t}^{2} / T_{\mathrm{FWHM}}{ }^{2}\right)$ is the envelope of the original pump pulse with peak power $P_{0}$ and duration $T_{\mathrm{FWHM}}$. The intensity modulation contrast (visibility) is given by $2 a_{0} /\left(1+a_{0}^{2}\right)$. This type of dual frequency field can be readily generated using optical frequency shifting techniques.

In the presence of significant modulation contrast, the input pulse can be considered as a series of ultrashort sub-pulses of duration $T_{\mathrm{i}} \sim 1 / 2 f_{\mathrm{m}}$ superimposed onto a broad lower amplitude background envelope. Under these conditions, the supercontinuum spectral broadening is dominated by the dynamics of these ultrashort sub-pulses, with only a minor contribution from the background envelope. We consider the case of pumping in the anomalous dispersion regime. To determine conditions on the modulation characteristics associated with coherent SC generation, we adapt previouslydeveloped criteria in the single pulse regime to the individual sub-pulses of the modulated envelope. In particular, if we consider the central sub-pulse of peak-to-background amplitude $P_{\mathrm{i}}=4 a_{0} P_{0}$, and soliton order $N_{\mathrm{i}}=\left(\gamma \mathrm{P}_{\mathrm{i}} \mathrm{T}_{\mathrm{i}} /\left|\beta_{2}\right|\right)^{1 / 2}$ we can expect coherent SC seeded by this sub-pulse provided $N_{\mathrm{i}}<N_{\text {cr }}$ where $N_{\mathrm{cr}} \sim 10$ generally ensures coherent SC. This condition, which applies for the case of anomalous dispersion regime pumping, can be recast into a more useful form in terms of the initial modulation frequency

$$
f_{m} \geq \frac{\sqrt{a_{0}}}{T_{F W H M}} \cdot \frac{N}{N_{c r}},
$$

and by requiring that the propagation length is less than the fission length of the central sub-pulse, we can estimate a useful guideline for the optimal fiber length

$$
L \approx \frac{1}{4 f_{m} \sqrt{2 \gamma a_{0} P_{0}\left|\beta_{2}\right|}} .
$$


In all of these results, $N$ is the input soliton number of the unmodulated pulse and $\beta_{2}$ and $\gamma$ represent the dispersion and nonlinearity at the pump wavelength. Although these results are based on the central sub-pulse parameters, of course neighboring sub-pulses would be expected to themselves induce similar coherent SC generation, with their presence manifested in the overall output spectrum in the form of spectral channeling at the frequency $f_{\mathrm{m}}$.

\subsection{Numerical modelling}

Although based on straightforward physical considerations, we have found using numerical simulations that the guidelines above accurately predict conditions coherent SC generation in the long pulse regime using picosecond pulses. Specifically, we consider the propagation of Gaussian $2 \mathrm{ps,} 6 \mathrm{~kW}$ peak power pulses at $1060 \mathrm{~nm}$ in a photonic crystal fiber with a zero-dispersion wavelength at $1025 \mathrm{~nm}$. The dispersion coefficients at the $1060 \mathrm{~nm}$ pump wavelength are: $\beta_{2}=-4.10 \mathrm{ps}^{2} \mathrm{~km}^{-1}, \beta_{3}=6.87 \times 10^{-2} \mathrm{ps}^{3} \mathrm{~km}^{-1}, \beta_{4}=-9.29 \times 10^{-3} \mathrm{ps}^{4} \mathrm{~km}^{-1}, \beta_{5}=2.45 \times 10^{-7} \mathrm{ps}^{5} \mathrm{~km}^{-1}, \beta_{6}=-9.79 \times 10^{-10} \mathrm{~s}^{6}$ $\mathrm{km}^{-1}, \beta_{7}=3.95 \times 10^{-12} \mathrm{ps}^{7} \mathrm{~km}^{-1}, \beta_{8}=-1.12 \times 10^{-14} \mathrm{ps}^{8} \mathrm{~km}^{-1}, \beta_{9}=1.90 \times 10^{-17} \mathrm{ps}^{9} \mathrm{~km}^{-1}, \beta_{10}=-1.51 \times 10^{-20} \mathrm{ps}^{10} \mathrm{~km}^{-1}$. The nonlinear coefficient is $\gamma=0.015 \mathrm{~W}^{-1} \mathrm{~m}^{-1}$ and $\tau_{\text {shock }}=0.66 \mathrm{fs}$. The input pulse parameters correspond to a soliton number $N$ $=177$ and under these conditions, one would expect strongly incoherent SC generation seeded by initial modulation instability dynamics. To model SC generation we use the same generalized nonlinear Schrödinger equation as in Section 2. We carry out multiple simulations using different noise seeds in order to determine average spectral characteristics and coherence properties.

Figure 7 illustrates the spectral and coherence characteristics of the generated SC after $20 \mathrm{~cm}$ in the absence of modulation on the input pulse yet in the presence of quantum noise. Clearly, the noise-seeded MI initial dynamics leads to a SC with large spectral variations from shot-to-shot and poor stability. The apparent smoothness is simply caused by the average over multiple shots.
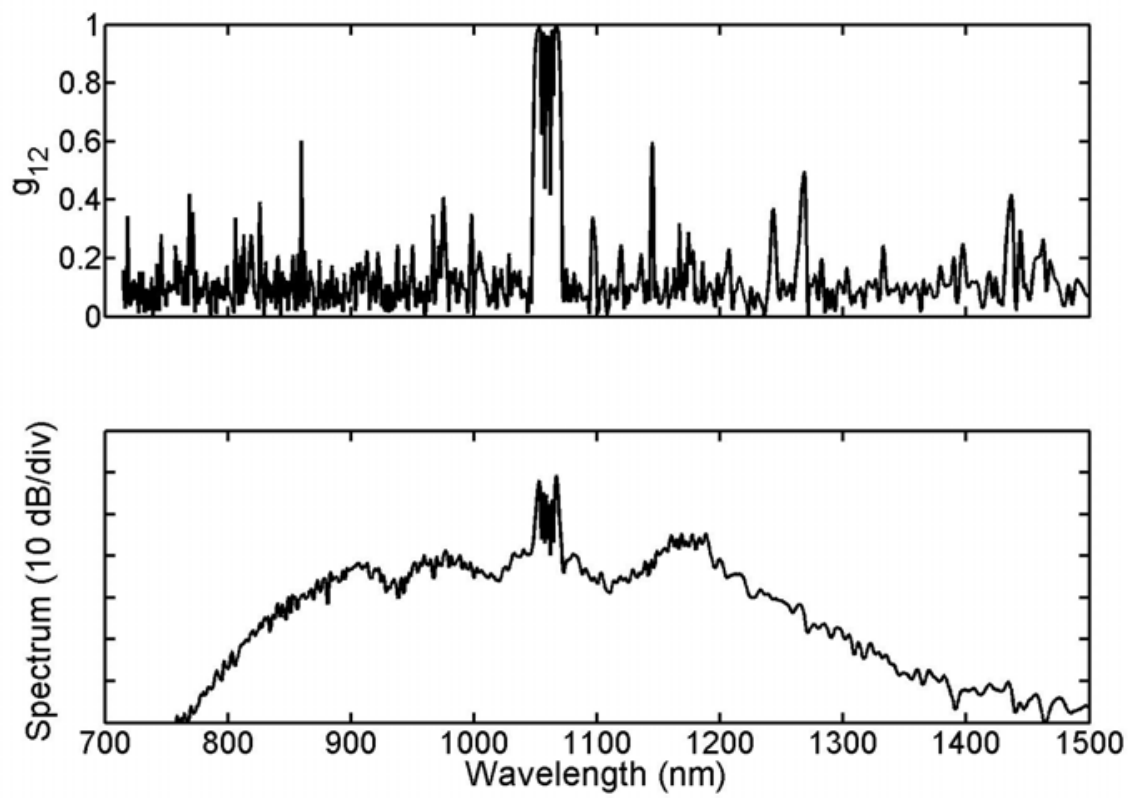

Fig. 7 Top: coherence and bottom: averaged SC spectrum generated in the unmodulated case.

We next impose a modulation on top of the input pulse with $a_{0}=0.15$ which ensures that the central sub-pulse contribution to the SC spectrum is significant. This value of $a_{0}$ gives a minimum value of $3.5 \mathrm{THz}$ for the modulation frequency for coherent SC generation. We choose $f_{\mathrm{m}}=5 \mathrm{THz}$ and a fiber length $L=12 \mathrm{~cm}$. Figure 8 shows the SC spectrum and associated coherence when the input pulse is modulated with the above characteristics. In comparison with the unmodulated pump case the SC spectrum exhibits a dramatic increase in coherence even though the overall bandwidth is comparable. This result is remarkable for the pulse duration and level of peak power envisaged here and to our knowledge represents the first example of how coherent SC can be generated in such a long pulse regime. Additional numerical simulations show that the SC coherence remains high across the SC bandwidth for longer fiber 
lengths exceeding several times the minimum value of $12 \mathrm{~cm}$ considered here. In general, we find that increasing $a_{0}$ improves further the stability which is expected as larger modulation contrast reduces the influence of the broad temporal background and is closer to the ideal case of a train of individual short pulses.
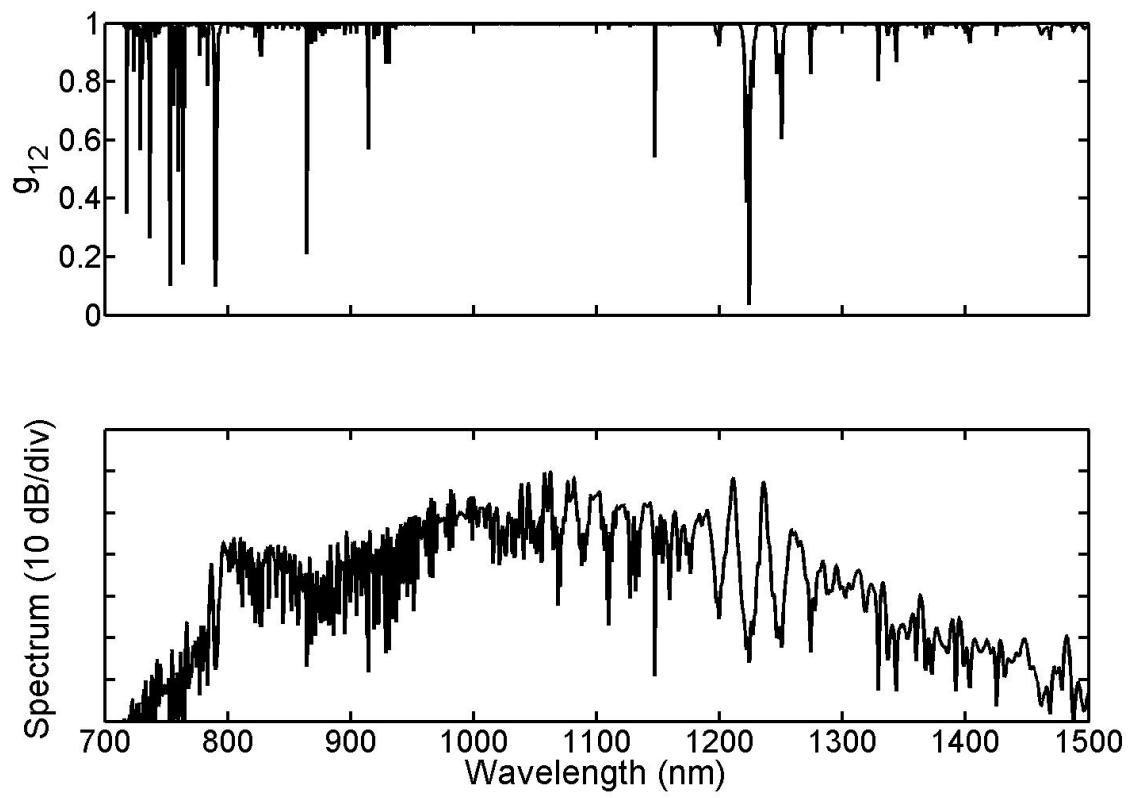

Fig. 8 Top: coherence and bottom: averaged SC spectrum generated when the input pulse is modulated.

\section{CONCLUSIONS}

We have investigated the possibility of generating broadband supercontinuum with high degree of coherence in the long pulse regime. Specifically, we have investigated the possibility of using fibers with a longitudinally-varying convex dispersion profile that allows to generate octave-spanning and relative flat supercontinuum spectra through efficient higher-order soliton compression and dispersive wave generation. Furthermore, we have shown that imposing an initial modulation with appropriate characteristics on top of the broad pulse can lead to a fully coherent continuum which is in marked contrast with incoherent spectra generated by unmodulated pulses. In fact, the recipes given here for the generation of coherent SC in the long pulse regime are rather general and can be further applied to even much longer initial pulse duration. These findings could help solving the long-standing problem of coherent SC generation in the long pulse regime.

\section{REFERENCES}

[1] J. K. Ranka, R. S. Windeler, and A. J. Stentz, "Visible continuum generation in air-silica microstructure optical fibers with anomalous dispersion at $800 \mathrm{~nm}$," Opt. Lett. 25, 25-27 (2000).

[2] A. V. Husakou and J. Herrmann, "Supercontinuum Generation of Higher-Order Solitons by Fission in Photonic Crystal Fibers," Phys. Rev. Lett. 87, 203 901/1-4 (2001).

[3] A. L. Gaeta, "Nonlinear Propagation and Continuum Generation in Microstructured Optical Fibers," Opt. Lett. 27, 924-926 (2002).

[4] K. L. Corwin, N. R. Newbury, J. M. Dudley, S. Coen, S. A. Diddams, K. Weber, and R. S. Windeler, "Fundamental Noise Limitations to Supercontinuum Generation in Microstructure Fiber," Phys. Rev. Lett. 90, 113 904/1-4 (2003).

[5] J. M. Dudley, G. Genty, and S. Coen, "Supercontinuum Generation in Photonic Crystal Fiber," Rev. Mod. Phys. 78, 1135-1184 (2006). 
M. N. Islam, G. Sucha, I. Bar-Joseph, M. Wegener, J. P. Gordon, and D. S. Chemla, "Broad Bandwidths from Frequency-Shifting Solitons in Fibers," Opt. Lett. 14, 370-372 (1989).

[7] M. N. Islam, G. Sucha, I. Bar-Joseph, M. Wegener, J. P. Gordon, and D. S. Chemla, "Femtosecond Distributed Soliton Spectrum in Fibers,” J. Opt. Soc. Am. B 6, 1149-1158 (1989).

[8] F. Vanholsbeeck, S. Martın-Lopez, M. Gonzalez-Herraez, and S. Coen, "The Role of Pump Incoherence in Continuous-Wave Supercontinuum Generation," Opt. Express , 6615-6625 (2005).

[9] S. M. Kobtsev, S. V. Kukarin, N. V. Fateev, and S. V. Smirnov, "Coherent, polarization and temporal properties of self-frequency shifted solitons generated in polarization-maintaining microstructured fibre," Appl. Phys. B 81, 265269 (2005).

[10] J. N. Kutz, C. Lynga, and B. J. Eggleton, "Enhanced Supercontinuum Generation Through DispersionManagement," Opt. Express 13, 3989-3998 (2005).

[11] M. H. Frosz, O. Bang, and A. Bjarklev, "Soliton collision and Raman gain regimes in continuous-wave pumped supercontinuum generation," Opt. Express 14, 9391-9407 (2006).

[12] A. Demircan and U. Bandelow, "Analysis of the interplay between soliton fission and modulation instability in supercontinuum generation," Appl. Phys. B 86, 31-39 (2007).

[13] B. A. Cumberland, J. C. Travers, S. V. Popov, and J. R. Taylor, "29 W High power CW supercontinuum source," Opt. Express 16, 5954-5962 (2008).

[14] D. R. Solli, C. Ropers, P. Koonath, and B. Jalali, "Optical rogue waves," Nature 450, 1054-1058 (2007).

[15] J. M. Dudley, G. Genty, and B. J. Eggleton, "Harnessing and control of optical rogue waves in supercontinuum generation," Opt. Express 16, 3644-3651 (2008).

[16] G. Genty, J.M. Dudley, B.J. Eggleton, "Modulation control and spectral shaping of optical fiber supercontinuum generation in the picosecond regime," Appl. Phys. B published online, 6 November 2008, DOI 10.1007/s00340-0083274-1 (2008).

[17] A. V. Shipulin, D. G. Fursa, E. A. Golovchenko, and E. M. Dianov, "High repetition rate cw fundamental soliton generation using multisoliton pulse compression in a varying dispersion fibre," Electron. Lett. 29, 1401-1403 (1993).

[18] T. Morioka, S. Kawanishi, K. Mori, and M. Saruwatari, "Nearly penalty-free, < 4 ps supercontinuum Gbit/s pulse generation over 1535-1560 nm," Electron. Lett. 30, 790-791 (1994).

[19] T. Morioka, S. Kawanishi, H. Takara, and O. Kamatani, "Penalty-free, 100Gbit/s optical transmission of less-than2ps supercontinuum transform-limited pulses over 40 km," Electron. Lett. 31, 124-125 (1995).

[20] T. Morioka, K. Uchiyama, S. Kawanishi, S. Suzuki, and M. Saruwatari, "Multiwavelength picosecond pulse source with low jitter and high optical frequency stability based on $200 \mathrm{~nm}$ supercontinuum filtering," Electron. Lett. 31, 1064-1066 (1995).

[21] K. Mori, T. Morioka, and M. Saruwatari, "Ultra wide spectral range group-velocity dispersion measurement utilizing supercontinuum in an optical fiber pumped by a $1.5 \mu \mathrm{m}$ compact laser source," IEEE Trans. Instrum. Meas. 44, 712-715 (1995).

[22] K. Mori, H. Takara, S. Kawanishi, M. Saruwatari, and T. Morioka, "Flatly broadened supercontinuum spectrum generated in a dispersion decreasing fibre with convex dispersion profile," Electron. Lett. 33, 1806-1808 (1997).

[23] K. Mori, H. Takara, and S. Kawanishi, "Analysis and design of supercontinuum pulse generation in a single-mode optical fiber," J. Opt. Soc. Am. B 18, 1780-1792 (2001).

[24] G. Genty, S. Coen, and J. Dudley, "Fiber supercontinuum sources," J. Opt. Soc. Am. B 24, 1771-1785 (2007).

[25] M. Monerie, "Propagation in doubly-clad single mode fiers," IEEE J. Quantum Electron. 18, 535-542 (1983).

[26] P. V. Mamyshev, P. G. J. Wigley, J. Wilson, G. I. Stegeman, V. A. Semenov, E. M. Dianov, and S. I. Miroshnichenko, "Adiabatic compression of Schrodinger solitons due to the combined perturbations of higher-order dispersion and delayed nonlinear response," Phys. Rev. Lett. 71, 73-76 (1993).

[27] B. Kibler, J. M. Dudley, and S. Coen, "Supercontinuum generation and nonlinear pulse propagation in photonic crystal fiber: influence of the frequency-dependent effective mode area," Appl. Phys. B 81, 337-342 (2005).

[28] H. H. Kuehl, "Solitons on an axially nonuniform optical fiber," J. Opt. Soc. Am. B 5, 709-713 (1988).

[29] S. V. Chernikov, E. M. Dianov, D. J. Richardson, and D. N. Payne, "Soliton pulse compression in dispersiondecreasing fiber," Opt. Lett. 18, 476-478 (1993).

[30] M. D. Pelusi and H. F. Liu, "Higher order soliton pulse compression in dispersion-decreasing optical fibers," IEEE J. Quantum Electron. 33, 1430-1439 (1997).

[31] E. B. Treacy, "Measurement and interpretation of dynamic spectrograms of picosecond light pulses," J. Appl. Phys. 42, 3848-3858 (1971). 
[32] G. Chang, T. B. Norris, and H. G. Winful, "Optimization of supercontinuum generation in photonic crystal fibers for pulse compression," Opt. Lett. 28, 546-548 (2003).

[33] J. M. Dudley and S. Coen, "Fundamental limits to few-cycle pulse generation from compression of supercontinuum spectra generated in photonic crystal fiber," Opt. Express 12, 2423-2428 (2004).

[34] J. Hu, B. S. Marks, C. R. Menyuk, J. Kim, T. F. Carruthers, B. M. Wright, T. T. F., and E. J. Friebele, "Pulse compression using a tapered microstructure optical fiber," Opt. Express 14, 4026-4036 (2006). 